\title{
Hamiltonians with two degrees of freedom admitting a singlevalued general solution*
}

\author{
Robert CONTE ${ }^{\dagger}$, Micheline MUSETTE M $^{\ddagger}$ and Caroline VERHOEVEN $\ddagger$ \\ †Service de physique de l'état condensé (URA 2464), CEA-Saclay \\ F-91191 Gif-sur-Yvette Cedex, France \\ E-mail: Conte@drecam.saclay.cea.fr \\ $\ddagger$ Dienst Theoretische Natuurkunde, Vrije Universiteit Brussel and \\ International Solvay Institutes for Physics and Chemistry \\ Pleinlaan 2, B-1050 Brussels, Belgium \\ E-mail: MMusette@vub.ac.be,CVerhoev@vub.ac.be
}

22 March 2005

\begin{abstract}
Following the basic principles stated by Painlevé, we first revisit the process of selecting the admissible time-independent Hamiltonians $H=\left(p_{1}^{2}+p_{2}^{2}\right) / 2+V\left(q_{1}, q_{2}\right)$ whose some integer power $q_{j}^{n_{j}}(t)$ of the general solution is a singlevalued function of the complex time $t$. In addition to the well known rational potentials $V$ of Hénon-Heiles, this selects possible cases with a trigonometric dependence of $V$ on $q_{j}$. Then, by establishing the relevant confluences, we restrict the question of the explicit integration of the seven (three "cubic" plus four "quartic") rational Hénon-Heiles cases to the quartic cases. Finally, we perform the explicit integration of the quartic cases, thus proving that the seven rational cases have a meromorphic general solution explicitly given by a genus two hyperelliptic function.
\end{abstract}

Keywords: two degree of freedom Hamiltonians, Painlevé test, Painlevé property, Hénon-Heiles Hamiltonian, hyperelliptic.

PACS 1995: 02.30.Hq, 03.40

\section{Contents}

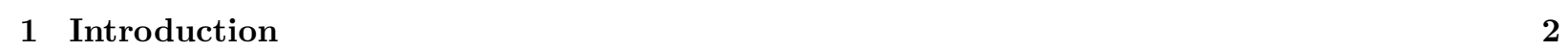

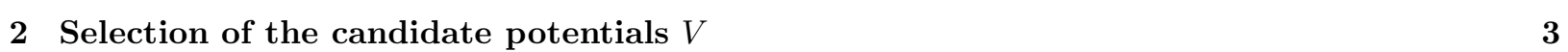

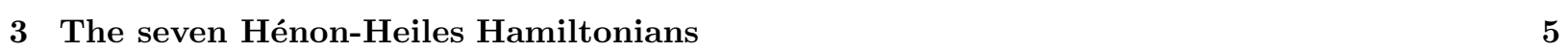

\begin{tabular}{|l|l}
\hline 4 Confluences from $\mathrm{HH} 4$ to $\mathrm{HH} 3$ & 7
\end{tabular}

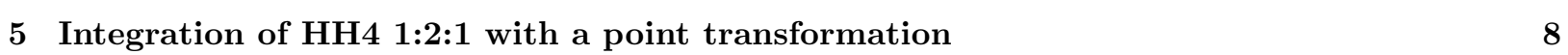

6 Integration of the 1:6:1, 1:6:8, 1:12:16 cases with birational transformations 9

7 Conclusion $\quad 9$

*Analysis in theory and applications (Nanjing), to appear, 2005. Nanjing, 20-24 July 2004. Corresponding author RC. Preprint S2005/006. nlin.SI/0507012 


\section{Introduction}

We consider the most general two-degree of freedom, classical, time-independent Hamiltonian of the physical type (i.e. the sum of a kinetic energy and a potential energy),

$$
H=\frac{1}{2}\left(p_{1}^{2}+p_{2}^{2}\right)+V\left(q_{1}, q_{2}\right)
$$

and the problem which we address is to determine all the potentials $V\left(q_{1}, q_{2}\right)$ such that some unspecified integer power $q_{j}^{n_{j}}(t)$ of the general solution is a single valued function of the complex time $t$.

In the case of one degree of freedom, this problem only admits two solutions,

$$
\begin{aligned}
& n= \pm 1, H=\frac{p^{2}}{2}+\sum_{j=1}^{4} c_{j} q^{j}, \\
& n= \pm 2, H=\frac{p^{2}}{2}+a q^{-2}+c_{2} q^{2}+c_{4} q^{4}+c_{6} q^{6} .
\end{aligned}
$$

In both cases, $q^{n}$ is an elliptic function and, in the second case, $q$ is generically multivalued.

This property that the general solution of a differential equation is singlevalued, except maybe at the singularities of the equation itself, is called the Painlevé property (PP) 4].

The equations of motion for $q_{j}(t)$ are obtained by eliminating the momenta $p_{1}, p_{2}$ between the Hamilton's equations of motion,

$$
\frac{\mathrm{d} p_{j}}{\mathrm{~d} t}=-\frac{\partial V\left(q_{1}, q_{2}\right)}{\partial q_{j}}, \frac{\mathrm{d} q_{j}}{\mathrm{~d} t}=q_{j}^{\prime}=p_{j}, j=1,2
$$

which results into the system of two coupled second order ordinary differential equations (ODE)

$$
q_{j}^{\prime \prime}+\frac{\partial V\left(q_{1}, q_{2}\right)}{\partial q_{j}}=0, j=1,2
$$

together with the first integral

$$
H \equiv \frac{q_{1}^{\prime 2}}{2}+\frac{q_{2}^{\prime 2}}{2}+V\left(q_{1}, q_{2}\right)=E .
$$

To prove the Painlevé property, one must perform the two following steps.

1. Generation of necessary conditions for the single valuedness of the general solution. This step is algorithmic and known as the Painlevé test [4. However, its output is only a set of necessary conditions, in our case a selection of candidate potentials $V\left(q_{1}, q_{2}\right)$.

2. For each such candidate $V$, explicit integration of the equations of motion, so as to indeed check the single valuedness of the general solution.

In section 2 we select the potentials $V\left(q_{1}, q_{2}\right)$ according to the prescriptions of the Painlevé test. In section 3 we present the seven so-called Hénon-Heiles Hamiltonians. In section 4 we establish confluences from one subset of these seven Hamiltonians to another subset, thus restricting the question of their explicit integration to the first subset. In sections 5 and 6 we recall the explicit integration of this first subset, the so-called "quartic" cases. 


\section{Selection of the candidate potentials $V$}

The difficulty is that very few results exist concerning coupled systems of nonlinear ODEs possessing the Painlevé property. On the contrary, for a single ODE, many results exist, either as exhaustive lists of equations in a given class (e.g. second order first degree) which possess the PP, or as precise necessary conditions to be satisfied.

Let us therefore build, by elimination of $q_{2}$, a single ODE in $q_{1}(t)$ in a class at least partially studied. Taking the shorthand notation

$$
V_{m, n}=\frac{\partial^{m+n} V\left(q_{1}, q_{2}\right)}{\partial q_{1}^{m} \partial q_{2}^{n}},
$$

one eliminates $q_{1}^{\prime \prime}, q_{2}^{\prime \prime}, q_{2}^{\prime}$ between the system of four equations made of (5) and the first two derivatives of

$$
q_{1}^{\prime \prime}+V_{10}\left(q_{1}, q_{2}\right)=0 .
$$

This yields

$$
\left\{\begin{array}{l}
q_{1}^{\prime \prime}=-V_{10}\left(q_{1}, q_{2}\right), \\
q_{2}^{\prime \prime}=-V_{01}\left(q_{1}, q_{2}\right), \\
q_{2}^{\prime}=-\frac{q_{1}^{\prime \prime}}{V_{11}}-\frac{V_{20}}{V_{11}} q_{1}^{\prime},
\end{array}\right.
$$

and the fourth order first degree ODE for $q_{1}(t)$

$$
-q_{1}^{\prime \prime \prime \prime}-V_{12}\left(\frac{q_{1}^{\prime \prime \prime}}{V_{11}}+\frac{V_{20}}{V_{11}} q_{1}^{\prime}\right)^{2}+2 V_{21}\left(\frac{q_{1}^{\prime \prime \prime}}{V_{11}}+\frac{V_{20}}{V_{11}} q_{1}^{\prime}\right) q_{1}^{\prime}-V_{20} q_{1}^{\prime \prime}-V_{30} q_{1}^{\prime 2}+V_{01} V_{11}=0,
$$

in which the coefficients $V_{m n}$ only depend on $\left(q_{1}^{\prime \prime}, q_{1}\right)$ after the (implicit) elimination of $q_{2}$ from (8).

The similar elimination with (6) yields the third order second degree ODE

$$
\frac{q_{1}^{\prime 2}}{2}+\frac{1}{2}\left(\frac{q_{1}^{\prime \prime \prime}}{V_{11}}+\frac{V_{20}}{V_{11}} q_{1}^{\prime}\right)^{2}+V-E=0 .
$$

None of the two ODEs (10), (11) is very helpful to generate necessary conditions on $V$, but the combination which eliminates $q_{1}^{\prime \prime \prime}$, namely

$$
-q_{1}^{\prime \prime \prime \prime}+2 \frac{V_{21}}{V_{11}} q_{1}^{\prime} q_{1}^{\prime \prime \prime}-V_{20} q_{1}^{\prime \prime}+\left(2 \frac{V_{21} V_{20}}{V_{11}}-V_{30}+V_{12}\right) q_{1}^{\prime 2}+V_{01} V_{11}+2(V-E) V_{12}=0,
$$

is quite helpful since it has only degree one in $q_{1}^{\prime \prime \prime}$.

Indeed, in 1902 Painlevé [18, p. 74] established necessary conditions for an $n$-th order first degree ODE

$$
u^{(n)}=F\left(u^{(n-1)}, \ldots, u, t\right),
$$

to possess the PP, when $F$ is assumed rational in $u^{(n-1)}, u^{(n-2)}$, algebraic in $u^{(n-3)}, \ldots, u$, and analytic in $t$ (we will also assume such a dependence for (12)).

The first necessary condition is that the highest derivative $u^{(n)}$, as a function of the next highest derivative $u^{(n-1)}$, be a polynomial of degree at most two (i.e. that the ODE for $u^{(n-1)}$ be of Riccati type),

$$
u^{(n)}=\sum_{j=0}^{2} A_{j}\left(u^{(n-2)}, \ldots, u, t\right)\left(u^{(n-1)}\right)^{j},
$$

which is indeed the case for both (10) and (12). 
The second necessary condition states that, as a function of the second next highest derivative $u^{(n-2)}$, each coefficient $A_{j}$ has for only singularities simple poles, the poles of $A_{1}$ and $A_{0}$ are among those of $A_{2}$, and the difference between the degrees of the numerator and denominator of $A_{j}$ does not exceed $-1,1,3$ for, respectively, $j=2,1,0$. When applied to (12), since $A_{2}$ is identically zero and thus has no poles (this feature is precisely the advantage of (12) over (10), this latter condition requires that the coefficients $A_{1} \equiv 2\left(V_{21} / V_{11}\right) q_{1}^{\prime}$ and $A_{0}$ (in which, as always, $q_{2}$ is eliminated from (8)) be polynomials in $q_{1}^{\prime \prime}$ with maximal respective degrees 1 and 3 . The necessary condition arising from $A_{1}$ is

$$
\exists F_{1}, G_{1}: \frac{V_{21}}{V_{11}}=F_{1}\left(q_{1}\right) q_{1}^{\prime \prime}+G_{1}\left(q_{1}\right) .
$$

Assuming the additional condition $F_{1}\left(q_{1}\right)=0$, the partial differential equation (15) is integrated as

$$
V\left(q_{1}, q_{2}\right)=f_{1}\left(q_{1}\right) f_{2}\left(q_{2}\right)+h_{1}\left(q_{1}\right)+h_{2}\left(q_{2}\right), f_{1}^{\prime} f_{2}^{\prime} \neq 0
$$

in which the four functions must be further constrained.

Instead of $f_{2}\left(q_{2}\right)$, let us introduce its inverse function $F_{2}\left(r_{1}\right)$ from (8),

$$
r_{1}=f_{2}\left(q_{2}\right), q_{2}=F_{2}\left(r_{1}\right), r_{1}=-\frac{q_{1}^{\prime \prime}-h_{1}^{\prime}\left(q_{1}\right)}{f_{1}^{\prime}\left(q_{1}\right)}
$$

which implies

$$
f_{2}^{\prime}\left(q_{2}\right)=\frac{1}{F_{2}^{\prime}\left(r_{1}\right)}, f_{2}^{\prime \prime}\left(q_{2}\right)=-\frac{F_{2}^{\prime \prime}\left(r_{1}\right)}{\left(F_{2}^{\prime}\left(r_{1}\right)\right)^{3}}
$$

The equation (12) then becomes

$$
\begin{aligned}
-q_{1}^{\prime \prime \prime \prime} & +\frac{f_{1}^{\prime \prime}}{f_{1}^{\prime}}\left(2 q_{1}^{\prime} q_{1}^{\prime \prime \prime}+q_{1}^{\prime \prime 2}\right)+\left(2 \frac{f_{1}^{\prime \prime \prime}}{f_{1}^{\prime}}-\frac{f_{1}^{\prime \prime 2}}{f_{1}^{\prime 2}}\right) q_{1}^{\prime 2} q_{1}^{\prime \prime} \\
& +\left(\frac{f_{1}^{\prime \prime} h_{1}^{\prime}}{f_{1}^{\prime}}-h_{1}^{\prime \prime}\right) q_{1}^{\prime \prime}+\left(\frac{f_{1}^{\prime \prime \prime}}{f_{1}^{\prime}} h_{1}^{\prime}+2 \frac{f_{1}^{\prime \prime}}{f_{1}^{\prime}} h_{1}^{\prime \prime}-h_{1}^{\prime \prime \prime}-2 \frac{f_{1}^{\prime \prime 2}}{f_{1}^{\prime 2}} h_{1}^{\prime}\right) q_{1}^{\prime 2} \\
& -\left(f_{1}^{\prime} q_{1}^{\prime 2}-2 f_{1} q_{1}^{\prime \prime}-2 E f_{1}^{\prime}+2\left(h_{1} f_{1}^{\prime}-h_{1}^{\prime} f_{1}\right)\right) \frac{F_{2}^{\prime \prime}\left(r_{1}\right)}{\left(F_{2}^{\prime}\left(r_{1}\right)\right)^{3}} \\
& +\frac{f_{1} f_{1}^{\prime}}{\left(F_{2}^{\prime}\left(r_{1}\right)\right)^{2}}+f_{1}^{\prime} \frac{\mathrm{d}}{\mathrm{d} r_{1}}\left(\left(F_{2}^{\prime}\left(r_{1}\right)\right)^{-2} h_{2}\right)=0
\end{aligned}
$$

in which the dependence on $q_{1}^{\prime \prime}$ is also implicit through the dependence on $r_{1}$ in the last two lines, and $A_{0}$ must be a polynomial in $q_{1}^{\prime \prime}$ of degree at most three.

The term $q_{1}^{\prime 2}$ first constrains $F_{2}$,

$$
\left(f_{2}^{\prime}\left(q_{2}\right)\right)^{2}=\frac{1}{\left(F_{2}^{\prime}\left(r_{1}\right)\right)^{2}}=P_{4}\left(r_{1}\right), f_{2}^{\prime \prime}\left(q_{2}\right)=-\frac{F_{2}^{\prime \prime}\left(r_{1}\right)}{\left(F_{2}^{\prime}\left(r_{1}\right)\right)^{3}}=\frac{1}{2} P_{4}^{\prime}\left(r_{1}\right), P_{4}\left(r_{1}\right)=\sum_{j=0}^{4} d_{j} r_{1}^{j}
$$

in which the coefficients $d_{j}$ are constant, then the term depending on $h_{2}$ generates the constraint

$$
h_{2}=\frac{Q_{5}\left(r_{1}\right)}{P_{4}\left(r_{1}\right)}, Q_{5}\left(r_{1}\right)=\sum_{j=0}^{5} e_{j} r_{1}^{j}
$$

in which the coefficients $e_{j}$ are constant. The resulting fourth power of $q_{1}^{\prime \prime}$,

$$
-5 \frac{e_{5}+d_{4} f_{1}}{f_{1}^{\prime 3}} q_{1}^{\prime \prime}
$$


must be canceled, which implies $d_{4}=0$ and $e_{5}=0$. Finally, if one performs the $\alpha$-transformation

$$
\left(t, q_{1}\right) \rightarrow\left(T, Q_{1}\right): t=\varepsilon T, q_{1}=a+\varepsilon Q_{1},
$$

the limit $\varepsilon \rightarrow 0$ of (19),

$$
-Q_{1}^{\prime \prime \prime \prime}+4 \frac{e_{4}+d_{3} f_{1}(a)}{\left(f_{1}^{\prime}(a)\right)^{2}} Q_{1}^{\prime \prime 3}=0,
$$

must have the $\mathrm{PP}$, which requires $d_{3}=0$ and $e_{4}=0$ since the constant $a$ is arbitrary.

The other $\alpha$-transformation

$$
\begin{aligned}
& \left(t, q_{1}\right) \rightarrow\left(T, Q_{1}\right): t=\varepsilon T, q_{1}=Q_{1}, \\
& -Q_{1}^{\prime \prime \prime \prime}+2 \frac{f_{1}^{\prime \prime}}{f_{1}^{\prime}} Q_{1}^{\prime} Q_{1}^{\prime \prime \prime}+\left(\frac{f_{1}^{\prime \prime}}{f_{1}^{\prime}}+3 d_{2} \frac{f_{1}^{\prime}}{f_{1}}+3 \frac{e_{3}}{f_{1}^{\prime}}\right) Q_{1}^{\prime 2}+\left(\frac{f_{1}^{\prime \prime \prime}}{f_{1}^{\prime}}-2 \frac{f_{1}^{\prime 2}}{f_{1}^{\prime 2}}-d_{2}\right) Q^{\prime 2} Q_{1}^{\prime \prime}=0,
\end{aligned}
$$

should constrain $f_{1}\left(q_{1}\right)$, but we have not further explored this way.

The differential equation obeyed by $f_{2}\left(q_{2}\right)$,

$$
\left(\frac{\mathrm{d} f_{2}}{\mathrm{~d} q_{2}}\right)^{2}=d_{0}+d_{1} f_{2}+d_{2} f_{2}^{2}
$$

has the Painlevé property, and its solutions are displayed in Table 1

Table 1: Admissible potentials $V\left(q_{1}, q_{2}\right)$ selected by the condition of singlevaluedness of $q_{1}(t)$. The potential must have the form (16), in which $f_{2}$ and $h_{2}$ have only four admissible values.

\begin{tabular}{|l|l|l|}
\hline$\left(d_{2}, d_{1}, d_{0}\right)$ & $f_{2}$ & Terms in $h_{2}$ \\
\hline$(\neq 0,0, \neq 0)$ & $a_{2} \sinh b_{2} q_{2}$ & $\ldots$ \\
\hline$(\neq 0,0,0)$ & $a_{2} e^{b_{2} q_{2}}$ & $\ldots$ \\
\hline$(0, \neq 0,0)$ & $a_{2} q_{2}^{2}+c_{2}$ & $q_{2}^{-2}, q_{2}^{2}, q_{2}^{4}$ \\
\hline$(0,0, \neq 0)$ & $b_{2} q_{2}$ & $q_{2}^{1}, q_{2}^{2}, q_{2}^{3}$ \\
\hline
\end{tabular}

The same study about the single valuedness of $q_{1}^{2}$ would lead to another, similar table listing a finite number of admissible potentials depending on a finite number of arbitrary constants. This Table 2 will be established in a very near future.

To conclude this first part of the Painlevé test, the admissible potentials $V$ for which the general solution $\left(q_{1}^{n_{1}}, q_{2}^{n_{2}}\right), n_{j}= \pm 1$ or \pm 2 , may be single valued are built by taking the appropriate information on $\left(f_{j}, h_{j}\right)$ from Tables 1 and 2] These potentials depend on a finite number of arbitrary constants.

The second part of the Painlevé test is very well known [17, 12] [4, §6.6] and consists in analyzing the system of two coupled second order ODEs (5), in order to enforce the absence of any branch point (either algebraic or logarithmic) whose location depends on the initial conditions (one says movable). This test is well defined only when the ODEs are algebraic, which is the probable reason for the usual discarding of the trigonometric cases. Although the test seems to have never been applied yet to the full rational cases isolated above, we will not perform here these lengthy calculations and directly skip to the question of the explicit integration of the candidate cases.

\section{The seven Hénon-Heiles Hamiltonians}

Among the set of rational potentials $V\left(q_{1}, q_{2}\right)$ selected in section 2 there exists a subset, called for historical reasons Hénon-Heiles Hamiltonians [14]. These seven potentials, usually denoted "cubic" or 
"quartic" according to their global degree in $\left(q_{1}, q_{2}\right)$, were in fact isolated by the condition that a second integral of the motion should exist [16] (Liouville integrability). The difference between the two approaches is quite important: requiring singlevaluedness generates necessary conditions on $V\left(q_{1}, q_{2}\right)$, while requiring the existence of a second integral of motion only results in sufficient conditions since $V\left(q_{1}, q_{2}\right)$ must be an input.

The cubic case basically arises from $f_{1}=b_{1} q_{1}, f_{2}=a_{2} q_{2}^{2}+c_{2}$, and the quartic case from $f_{1}=a_{1} q_{1}^{2}+$ $c_{1}, f_{2}=a_{2} q_{2}^{2}+c_{2}$, and their usual notation is as follows.

1. In the cubic case HH3 [3, 11,

$$
H=\frac{1}{2}\left(p_{1}^{2}+p_{2}^{2}+\omega_{1} q_{1}^{2}+\omega_{2} q_{2}^{2}\right)+\alpha q_{1} q_{2}^{2}-\frac{\beta}{3} q_{1}^{3}+\frac{\gamma}{2} q_{2}^{-2}, \alpha \neq 0
$$

in which the constants $\alpha, \beta, \omega_{1}, \omega_{2}$ and $\gamma$ can only take the three sets of values,

$$
\begin{aligned}
(\mathrm{SK}): & \beta / \alpha=-1, \omega_{1}=\omega_{2}, \\
(\mathrm{KdV} 5): & \beta / \alpha=-6, \\
(\mathrm{KK}): & \beta / \alpha=-16, \omega_{1}=16 \omega_{2} .
\end{aligned}
$$

2. In the quartic case $\mathrm{HH} 4$ [19, 13 ,

$$
\begin{aligned}
H= & \frac{1}{2}\left(P_{1}^{2}+P_{2}^{2}+\Omega_{1} Q_{1}^{2}+\Omega_{2} Q_{2}^{2}\right)+C Q_{1}^{4}+B Q_{1}^{2} Q_{2}^{2}+A Q_{2}^{4} \\
& +\frac{1}{2}\left(\frac{\alpha}{Q_{1}^{2}}+\frac{\beta}{Q_{2}^{2}}\right)+\gamma Q_{1}, B \neq 0,
\end{aligned}
$$

in which the constants $A, B, C, \alpha, \beta, \gamma, \Omega_{1}$ and $\Omega_{2}$ can only take the four values (the notation $A: B$ : $C=p: q: r$ stands for $A / p=B / q=C / r=$ arbitrary),

$$
\left\{\begin{array}{l}
A: B: C=1: 2: 1, \gamma=0, \\
A: B: C=1: 6: 1, \gamma=0, \Omega_{1}=\Omega_{2}, \\
A: B: C=1: 6: 8, \alpha=0, \Omega_{1}=4 \Omega_{2}, \\
A: B: C=1: 12: 16, \gamma=0, \Omega_{1}=4 \Omega_{2} .
\end{array}\right.
$$

For each of the seven cases so isolated there exists a second constant of the motion $K$ [10, 2, 15, [16, 1, 2] in involution with the Hamiltonian,

$$
\begin{aligned}
(\mathrm{SK}): K= & \left(3 p_{1} p_{2}+\alpha q_{2}\left(3 q_{1}^{2}+q_{2}^{2}\right)+3 \omega_{2} q_{1} q_{2}\right)^{2}+3 \gamma\left(3 p_{1}^{2} q_{2}^{-2}+4 \alpha q_{1}+2 \omega_{2}\right), \\
(\mathrm{KdV} 5): K= & 4 \alpha p_{2}\left(q_{2} p_{1}-q_{1} p_{2}\right)+\left(4 \omega_{2}-\omega_{1}\right)\left(p_{2}^{2}+\omega_{2} q_{2}^{2}+\gamma q_{2}^{-2}\right) \\
& +\alpha^{2} q_{2}^{2}\left(4 q_{1}^{2}+q_{2}^{2}\right)+4 \alpha q_{1}\left(\omega_{2} q_{2}^{2}-\gamma q_{2}^{-2}\right), \\
(\mathrm{KK}): K= & \left(3 p_{2}^{2}+3 \omega_{2} q_{2}^{2}+3 \gamma q_{2}^{-2}\right)^{2}+12 \alpha p_{2} q_{2}^{2}\left(3 q_{1} p_{2}-q_{2} p_{1}\right) \\
& -2 \alpha^{2} q_{2}^{4}\left(6 q_{1}^{2}+q_{2}^{2}\right)+12 \alpha q_{1}\left(-\omega_{2} q_{2}^{4}+\gamma\right)-12 \omega_{2} \gamma, \\
1: 2: 1: K= & \left(Q_{2} P_{1}-Q_{1} P_{2}\right)^{2}+Q_{2}^{2} \frac{\alpha}{Q_{1}^{2}}+Q_{1}^{2} \frac{\beta}{Q_{2}^{2}} \\
& -\frac{\Omega_{1}-\Omega_{2}}{2}\left(P_{1}^{2}-P_{2}^{2}+Q_{1}^{4}-Q_{2}^{4}+\Omega_{1} Q_{1}^{2}-\Omega_{2} Q_{2}^{2}+\frac{\alpha}{Q_{1}^{2}}-\frac{\beta}{Q_{2}^{2}}\right), A=\frac{1}{2}, \\
1: 6: 1: K= & \left(P_{1} P_{2}+Q_{1} Q_{2}\left(-\frac{Q_{1}^{2}+Q_{2}^{2}}{8}+\Omega_{1}\right)\right)^{2} \\
& -P_{2}^{2} \frac{\kappa_{1}^{2}}{Q_{1}^{2}}-P_{1}^{2} \frac{\kappa_{2}^{2}}{Q_{2}^{2}}+\frac{1}{4}\left(\kappa_{1}^{2} Q_{2}^{2}+\kappa_{2}^{2} Q_{1}^{2}\right)+\frac{\kappa_{1}^{2} \kappa_{2}^{2}}{Q_{1}^{2} Q_{2}^{2}}, \alpha=-\kappa_{1}^{2}, \beta=-\kappa_{2}^{2}, A=-\frac{1}{32},
\end{aligned}
$$




$$
\begin{aligned}
1: 6: 8: K= & \left(P_{2}^{2}-\frac{Q_{2}^{2}}{16}\left(2 Q_{2}^{2}+4 Q_{1}^{2}+\Omega_{2}\right)+\frac{\beta}{Q_{2}^{2}}\right)^{2}-\frac{1}{4} Q_{2}^{2}\left(Q_{2} P_{1}-2 Q_{1} P_{2}\right)^{2} \\
+ & \gamma\left(-2 \gamma Q_{2}^{2}-4 Q_{2} P_{1} P_{2}+\frac{1}{2} Q_{1} Q_{2}^{4}+Q_{1}^{3} Q_{2}^{2}+4 Q_{1} P_{2}^{2}-4 \Omega_{2} Q_{1} Q_{2}^{2}+4 Q_{1} \frac{\beta}{Q_{2}^{2}}\right), \\
& A=-\frac{1}{16}, \\
1: 12: 16: K= & \left(8\left(Q_{2} P_{1}-Q_{1} P_{2}\right) P_{2}-Q_{1} Q_{2}^{4}-2 Q_{1}^{3} Q_{2}^{2}+2 \Omega_{1} Q_{1} Q_{2}^{2}-8 Q_{1} \frac{\beta}{Q_{2}^{2}}\right)^{2} \\
& +\frac{32 \alpha}{5}\left(Q_{2}^{4}+10 \frac{Q_{2}^{2} P_{2}^{2}}{Q_{1}^{2}}\right), A=-\frac{1}{32} .
\end{aligned}
$$

\section{Confluences from $\mathrm{HH} 4$ to $\mathrm{HH} 3$}

As is well known, there exists a limiting process (confluence) which, starting from the Gauss hypergeometric equation, generates the sequence: Whittaker equation, Hermite-Weber and Bessel equations, Airy equation. The general solution of all these equations is therefore deductible from that of the Gauss hypergeometric equation.

A similar confluence also exists [20, 21, 17 among the seven HH Hamiltonians, and each cubic case can be obtained by a confluence of at least one quartic case.

The following confluences have been established,

$$
\left\{\begin{array}{l}
\text { HH4 1:2:1 } \rightarrow \text { HH3 KdV5, } \\
\text { HH4 1:6:8 } \rightarrow \text { HH3 KK, } \\
\text { HH4 1:6:8 } \rightarrow \text { HH3 KdV5, } \\
\text { HH4 1:12:16 } \rightarrow \text { HH3 KK. } \\
\text { HH4 1:12:16 } \rightarrow \text { HH3 SK. }
\end{array}\right.
$$

The absence of any confluence originating from HH4 1:6:1 still has to be explained.

Consider for instance the quartic 1:12:16,

$$
\left\{\begin{array}{l}
h_{1: 12: 16}(t)=\frac{1}{2}\left(p_{1}^{2}+p_{2}^{2}\right)+\frac{\omega}{8}\left(4 q_{1}^{2}+q_{2}^{2}\right)-\frac{n}{32}\left(16 q_{1}^{4}+12 q_{1}^{2} q_{2}^{2}+q_{2}^{4}\right) \\
\quad+\frac{1}{2}\left(\frac{\alpha}{q_{1}^{2}}+\frac{\beta}{q_{2}^{2}}\right) .
\end{array}\right.
$$

It admits a confluence to both the HH3 KK and SK cases,

$$
\left\{\begin{array}{l}
H_{\mathrm{KK}}(T)=\frac{1}{2}\left(P_{1}^{2}+P_{2}^{2}\right)+\frac{\Omega}{2}\left(16 Q_{1}^{2}+Q_{2}^{2}\right)+N\left(Q_{1} Q_{2}^{2}+\frac{16}{3} Q_{1}^{3}\right)+\frac{B}{2 Q_{2}^{2}}, \\
H_{\mathrm{SK}}(T)=\frac{1}{2}\left(P_{1}^{2}+P_{2}^{2}\right)+\frac{\Omega}{2}\left(Q_{1}^{2}+Q_{2}^{2}\right)+N\left(Q_{1} Q_{2}^{2}+\frac{1}{3} Q_{1}^{3}\right)+\frac{B}{2 Q_{2}^{2}},
\end{array}\right.
$$

they are (the integers $e_{t}$ and $e_{1}$ can be chosen arbitrarily),

$$
1: 12: 16 \rightarrow \mathrm{KK}\left\{\begin{array}{l}
t=\varepsilon^{e_{t}} T, q_{1}=\varepsilon^{e_{1}}\left(1+\varepsilon Q_{1}\right), q_{2}=\varepsilon^{1+e_{1}} Q_{2}, n=-\frac{4}{3} \varepsilon^{-2 e_{1}-2 e_{t}-1} N, \\
\alpha=\varepsilon^{4 e_{1}-2 e_{t}-1}\left(-\frac{4}{3} N+4 \Omega \varepsilon\right), \beta=\varepsilon^{4 e_{1}-2 e_{t}+4} B, \\
\omega=\varepsilon^{-2 e_{t}-1}(-4 N+4 \Omega \varepsilon), h=\varepsilon^{2 e_{1}-2 e_{t}-1}\left(-2 N+4 \Omega \varepsilon+H \varepsilon^{3}\right), \varepsilon \rightarrow 0,
\end{array}\right.
$$

and

$$
1: 12: 16 \rightarrow \mathrm{SK}\left\{\begin{array}{l}
t=\varepsilon^{e_{t}} T, q_{1}=\varepsilon^{e_{1}} Q_{2}, q_{2}=\varepsilon^{e_{1}-1}\left(1+\varepsilon Q_{1}\right), n=\varepsilon^{-2 e_{1}-2 e_{t}-1} N, \\
\alpha=\varepsilon^{4 e_{1}-2 e_{t}} B, \beta=\varepsilon^{4 e_{1}-2 e_{t}-5}\left(\frac{N}{16}+\frac{1}{4} \Omega \varepsilon\right), \\
\omega=\varepsilon^{-2 e_{t}-1}\left(\frac{3}{4} N+\Omega \varepsilon\right), h=\varepsilon^{2 e_{1}-2 e_{t}-3}\left(\frac{3}{32} N+\frac{1}{4} \Omega \varepsilon+H \varepsilon^{3}\right) .
\end{array}\right.
$$


One checks the loss of one parameter in the process, since the three quartic parameters $(\alpha, \beta, \omega)$ coalesce to only two cubic parameters $(B, \Omega)$.

From the quartic case HH4 1:2:1 to the cubic case HH3 KdV5, the confluence is

$$
1: 2: 1 \rightarrow \operatorname{KdV} 5\left\{\begin{array}{l}
h_{1: 2: 1}(t)=\frac{1}{2}\left(p_{1}^{2}+p_{2}^{2}\right)+\frac{\omega_{1}}{2} q_{1}^{2}+\frac{\omega_{2}}{2} q_{2}^{2}-\frac{n}{2}\left(q_{1}^{4}+2 q_{1}^{2} q_{2}^{2}+q_{2}^{4}\right)+\frac{1}{2}\left(\frac{\alpha}{q_{1}^{2}}+\frac{\beta}{q_{2}^{2}}\right) \\
H_{\mathrm{KdV} 5}(T)=\frac{1}{2}\left(P_{1}^{2}+P_{2}^{2}\right)+\frac{\Omega_{1}}{2} Q_{1}^{2}+\frac{\Omega_{2}}{2} Q_{2}^{2}-2 N\left(Q_{1} Q_{2}^{2}+2 Q_{1}^{3}\right)+\frac{B}{2 Q_{2}^{2}} \\
t=\varepsilon^{e_{t}} T, q_{1}=\varepsilon^{e_{1}}\left(1+\varepsilon Q_{1}\right), q_{2}=\varepsilon^{1+e_{1}} Q_{2}, n=\varepsilon^{-1-2 e_{1}-2 e_{t}} N, \\
\alpha=\varepsilon^{4 e_{1}-2 e_{t}-1}\left(N-\frac{\Omega_{1}}{12} \varepsilon\right), \beta=\varepsilon^{4 e_{1}-2 e_{t}+4} B, h=\varepsilon^{2 e_{1}-2 e_{t}-1}\left(\frac{3}{2} N+\frac{\Omega_{1}}{4} \varepsilon+H \varepsilon^{3}\right), \\
\omega_{1}=\varepsilon^{-2 e_{t}-1}\left(3 N+\frac{\Omega_{1}}{4} \varepsilon\right), \omega_{2}=\varepsilon^{-2 e_{t}-1}\left(2 N+\Omega_{2} \varepsilon\right) .
\end{array}\right.
$$

Since the three HH3 cases have been generated from some quartic case, it is useless to find the general solution of the cubic cases.

It is quite instructive to also perform the confluence starting from HH4 1:6:8. In fact, there exist two mutually exclusive subcases of 1:6:8 which are Liouville-integrable [16], these are

$$
\begin{array}{ll}
1: 6: 8 \mathrm{a} & H=(32), \alpha=0, \\
1: 6: 8 \mathrm{~b} & H=(32)+\frac{\nu}{q_{2}^{6}}, \gamma=0,
\end{array}
$$

and, if one requires the presence of the inverse square term $q_{1}^{-2}$ in the resulting HH3 case, only the subcase HH4 1:6:8b is able to achieve a confluence to a cubic case, and only two cubic cases can be produced: HH3 KK with an additional term $\nu_{\mathrm{KK}} q_{2}^{-6}$ (therefore also Liouville integrable [16]) and HH3 KdV5 provided $\nu$ is nonzero. With the definition

$$
\left\{\begin{array}{l}
h_{1: 6: 8 b}(t)=\frac{1}{2}\left(p_{1}^{2}+p_{2}^{2}\right)+\frac{\omega}{2}\left(4 q_{1}^{2}+q_{2}^{2}\right)-\frac{n}{16}\left(8 q_{1}^{4}+6 q_{1}^{2} q_{2}^{2}+q_{2}^{4}\right) \\
+\frac{1}{2}\left(\frac{\alpha}{q_{1}^{2}}+\frac{\beta}{q_{2}^{2}}\right)+\frac{\nu}{q_{2}^{6}}
\end{array}\right.
$$

the results are

$$
1: 6: 8 \mathrm{~b} \rightarrow \mathrm{KK}\left\{\begin{aligned}
t & =\varepsilon^{e_{t}} T, q_{1}=\varepsilon^{e_{1}}\left(1+\varepsilon Q_{1}\right), q_{2}=\varepsilon^{e_{1}+1} Q_{2}, n=\varepsilon^{-2 e_{1}-2 e_{t}-1} N, \\
\alpha & =\varepsilon^{4 e_{1}-2 e_{t}-1}\left(-\frac{4}{3} N+4 \Omega \varepsilon\right), \beta=\varepsilon^{4 e_{1}-2 e_{t}+1} B, \nu=\varepsilon^{8 e_{1}-2 e_{t}+8} \nu_{\mathrm{KK}}, \\
\omega & =\varepsilon^{-2 e_{t}-1}(-N+\Omega \varepsilon), h=\varepsilon^{2 e_{1}-2 e_{t}-1}\left(-2 N+4 \Omega \varepsilon+H \varepsilon^{2}\right),
\end{aligned}\right.
$$

and

$$
1: 6: 8 \mathrm{~b} \rightarrow \operatorname{KdV} 5\left\{\begin{aligned}
t & =\varepsilon^{e_{t}} T, q_{1}=\varepsilon^{e_{1}} Q_{2}, q_{2}=\varepsilon^{e_{1}-1}\left(1+\varepsilon Q_{1}\right), n=\varepsilon^{-2 e_{1}-2 e_{t}+1} N, \\
\alpha & =\varepsilon^{4 e_{1}-2 e_{t}} B, \beta=\varepsilon^{4 e_{1}-2 e_{t}-5}\left(-\frac{N}{4}+\frac{1}{4}\left(2 \Omega_{2}-\Omega_{1}\right) \varepsilon\right), \\
\nu & =\varepsilon^{8 e_{1}-2 e_{t}-9}\left(\frac{N}{32}+\frac{1}{24}\left(\Omega_{1}-\Omega_{2}\right) \varepsilon\right), \\
\omega & =\varepsilon^{-2 e_{t}-1}\left(\frac{3 N}{16}+\frac{\Omega_{2}}{4} \varepsilon\right), h=\varepsilon^{2 e_{1}-2 e_{t}-3}\left(-\frac{N}{16}+\frac{1}{12}\left(4 \Omega_{2}-\Omega_{1}\right) \varepsilon+H \varepsilon^{3}\right) .
\end{aligned}\right.
$$

\section{Integration of HH4 1:2:1 with a point transformation}

The two cases HH4 1:2:1 and HH3 KdV5 are related by the (one-way) confluence (50), but their relation is even stronger, and there exists a point transformation [6] Eq. (7.14)] between this quartic 1:2:1 case 
$H\left(Q_{j}, P_{j}, \Omega_{1}, \Omega_{2}, A, B\right)$ and the cubic $\operatorname{KdV} 5$ case $H\left(q_{j}, p_{j}, \omega_{1}, \omega_{2}, \alpha, \gamma\right)$.

$$
\left\{\begin{array}{l}
Q_{1}^{2}+Q_{2}^{2}+\frac{\Omega_{1}+\Omega_{2}}{5}=\alpha q_{1}+\frac{\omega_{1}+4 \omega_{2}}{20}, \\
\left(\Omega_{1}-\Omega_{2}\right)\left(Q_{1}^{2}-Q_{2}^{2}\right)=\frac{\alpha^{2}}{2} q_{2}^{2}-\frac{4 \omega_{1}+26 \omega_{2}}{5} \alpha q_{1}-\frac{\left(\omega_{1}+4 \omega_{2}\right)^{2}}{100}+2 E, \\
\Omega_{1}=\omega_{1}, \Omega_{2}=4 \omega_{2},
\end{array}\right.
$$

Its action on the genus two hyperelliptic curve which integrates KdV5 [10] is just a translation.

It is worthwhile to notice that the variables $Q_{1}$ and $Q_{2}$ of 1:2:1 and the variable $q_{2}$ of KdV5 are generically multivalued.

An attempt to find point transformations between the other quartic cases and any cubic case has been unsuccessful for the moment.

\section{Integration of the $1: 6: 1,1: 6: 8,1: 12: 16$ cases with birational transformations}

The classification of fourth order first degree ODEs in the class

$$
y^{\prime \prime \prime \prime}=P\left(y^{\prime \prime}, y^{\prime}, y ; t\right),
$$

in which $P$ is polynomial in $y^{\prime \prime}, y^{\prime}, y$ and analytic in $t$, has been recently completed [8]. Although this class is too restrictive to include our equation (19), there exist transformations [6] mapping each HH4 case to at least one time-independent equation with the PP in the class (57). These transformations, which are birational transformations, conserve the PP, therefore they establish the PP for all the quartic cases and, by confluence, for all the cubic cases. Their explicit form is not very compact, so we refer to Ref. [6] for further details.

\section{Conclusion}

Although we have not yet finished to revisit the derivation of all the two degree of freedom time-independent Hamiltonians with the Painlevé property, we thought it worthwhile to perform it starting from the basic principles, so as to avoid any a priori restriction on $V\left(q_{1}, q_{2}\right)$.

About the integration of the seven Hénon-Heiles Hamiltonians, the result, already summarized elsewhere [6], is the following. All these seven Hamiltonians have a meromorphic general solution, expressed with hyperelliptic functions of genus two, therefore they have the Painlevé property. Moreover, these seven Hamiltonians are complete in the Painlevé sense, i.e. it is impossible to add any time-independent term to the Hamiltonian without ruining the Painlevé property.

\section{Acknowledgments}

The authors acknowledge the financial support of the Tournesol grant no. T2003.09. RC warmly thanks the organizers for invitation. CV is a postdoctoral fellow at the FWO-Vlaanderen.

\section{References}

[1] S. Baker, Squared eigenfunction representations of integrable hierarchies, PhD Thesis, University of Leeds (1995). 
[2] S. Baker, V.Z. Enol'skii, and A. P. Fordy, Integrable quartic potentials and coupled KdV equations, Phys. Lett. A 201 (1995) 167-174.

[3] Chang Y. F., M. Tabor, and J. Weiss, Analytic structure of the Hénon-Heiles Hamiltonian in integrable and nonintegrable regimes, J. Math. Phys. 23 (1982) 531-538.

[4] R. Conte, The Painlevé approach to nonlinear ordinary differential equations, The Painlevé property, one century later, 77-180, ed. R. Conte, CRM series in mathematical physics (Springer, New York, 1999). solv-int/9710020

[5] R. Conte, A. P. Fordy, and A. Pickering, A perturbative Painlevé approach to nonlinear differential equations, Physica D 69 (1993) 33-58.

[6] R. Conte, M. Musette, and C. Verhoeven, Explicit integration of the Hénon-Heiles Hamiltonians, J. Nonlinear Mathematical Physics 12 Supp. 1 (2005) 212-227. Special issue in honor of F. Calogero. http://arxiv.org/abs/nlin.SI/0412057

[7] R. Conte, M. Musette, and C. Verhoeven, Completeness of the Hénon-Heiles Hamiltonians, Séminaires et congrès, in print (Société mathématique de France, Paris, 2005).

[8] C.M. Cosgrove, Higher order Painlevé equations in the polynomial class, I. Bureau symbol P2, Stud. Appl. Math. 104 (2000) 1-65.

[9] C.M. Cosgrove, Higher order Painlevé equations in the polynomial class, II. Bureau symbol P1, http://www.maths.usyd.edu.au:8000/res/Nonlinear/Cos/2000-6.html 113 pages, preprint 2000-6, University of Sydney (2000).

[10] J. Drach, Sur l'intégration par quadratures de l'équation $\frac{\mathrm{d}^{2} y}{\mathrm{~d} x^{2}}=[\varphi(x)+h] y$, C. R. Acad. Sc. Paris $168(1919)$ $337-340$.

[11] A. P. Fordy, The Hénon-Heiles system revisited, Physica D 52 (1991) 204-210.

[12] B. Gambier, Sur les équations différentielles du second ordre et du premier degré dont l'intégrale générale est à points critiques fixes, Acta Math. 33 (1910) 1-55.

[13] B. Grammaticos, B. Dorizzi, and A. Ramani, Integrability of Hamiltonians with third- and fourth-degree polynomial potentials, J. Math. Phys. 24 (1983) 2289-2295.

[14] M. Hénon and C. Heiles, The applicability of the third integral of motion: some numerical experiments, Astron. J. 69 (1964) $73-79$.

[15] J. Hietarinta, Classical versus quantum integrability, J. Math. Phys. 25 (1984) 1833-1840.

[16] J. Hietarinta, Direct method for the search of the second invariant, Phys. Rep. 147 (1987) 87-154.

[17] Sophie Kowalevski, Sur une propriété du système d'équations différentielles qui définit la rotation d'un corps solide autour d'un point fixe, Acta Math. 14 (1890) 81-93.

[18] P. Painlevé, Sur les équations différentielles du second ordre et d'ordre supérieur dont l'intégrale générale est uniforme, Acta Math. 25 (1902) 1-85.

[19] A. Ramani, B. Dorizzi, and B. Grammaticos, Painlevé conjecture revisited, Phys. Rev. Lett. 49 (1982) $1539-$ 1541.

[20] F.J. Romeiras, A note on integrable two-degrees-of-freedom Hamiltonian systems with a second integral quartic in the momenta, J. Phys. A 28 (1995) 5633-5642.

[21] C. Verhoeven, Integration of Hamiltonian systems of Hénon-Heiles type and their associated soliton equations, PhD thesis, Vrije Universiteit Brussel (May 2003). 